\title{
Measurement Science: Constructing Bridges between Reality and Knowledge
}

\author{
Luca Mari and Dario Petri
}

In this paper, we propose an explanation of the crucial role of measurement in our society by describing measurement as a bridge between the empirical world and the information world. We describe measurement science as built upon this feature, and we discuss its basic motivation and scope accordingly.

\section{Introduction}

Measurement is as old as civilization. It derives from counting, and it developed in the ancient world mainly through trade, astronomy, building, tools, and arms production. It was the basis for the geographical discoveries, the development of modern science, and the growth of industrialization. The social role of measurement and measurement standards is critical, as excellently presented in its historical perspective in [1]. Currently, measurement is not only a key discipline for scientific investigation, but is in all domains of human activities and endeavors to promote social evolution and prosperity. In advanced countries, measurements are pervasive in a huge number of situations in a multitude of subjects.

Measurement is in particular a key enabler for companies to compete globally in high-tech markets. Indeed, metrological infrastructures are an integral part of manufacturing processes and interface with all steps of product development, assuring production efficiency as well as product and service quality, which are both widely recognized as cornerstones of competitive success and business sustainability. In addition, in successful trading, business organizations employ regulatory frameworks, grounded on measurement confidence, ensuring access to global markets that are fair, open, and without unnecessary trade barriers. Measurement plays an increasingly important role in emerging technologies and innovations, thus enabling the development of new products, the creation of new industries, and improvement in productivity or in the quality of products and services. Conversely, emerging technologies offer new advancement opportunities to existing metrology due to demands for new instrumentation and measurement.

According to the principle that objective data are generally the basis of effective decisions [2], the management of entire organizations employs measurement. Measurement is then a key enabler in transportation, environmental protection, food industries, chemical industries, pharmaceutics, medical diagnosis and treatment, as well as social and psychological sciences. 
Thus, it is not surprising that currently "an estimated 80\% [of world trade] is affected by standards and regulations" and that, according to various studies, "the cost to producers and service providers of complying with standards can be $10 \%$ of production costs” [3].

Measurement is, of course, the basis to assess such compliance.

Unexpectedly, despite the high effectiveness of measurement for the progress of society and its pervasive practice in human activities, the nature and extent of knowledge pertaining to measurement are quite vague. As a result, generation of new knowledge about measurement frequently has little regard to where it is framed in the whole picture [4]: there is disregard of fundamental measurement concepts and presentations when teaching the discipline are stereotypical. Use of terminology is often peculiar, idiosyncratic, and based on implicit assumptions, and the bulk of practitioners do not make the best use of the available body of knowledge.

Various good reasons are responsible for this situation related to the organization of the measurement-related aspects of the scientific enterprise as shaped by historical evolution. Galileo emphasized the importance of the experimental method from which the industrial revolution received a decisive trigger: measurement then became the tool to obtain pure data from empirical phenomena, a critical role usually and implicitly assumed as unproblematic. “The results of measurement are neutral and precise; they cannot mislead" as T. S. Kuhn synthesized [5, p.165]. That paper was a turning point; it was a reflection on the actual function of measurement in a context where the "most prevalent notions both about the function of measurement and about the source of its special efficacy are derived largely from myth” [5, p.161].

However, most intellectual efforts on measurement are currently devoted to specific aspects of highly specialized technological topics, often seemingly ignoring the necessary systemic context that frames them. In sharp contrast, it is becoming steadily clearer that the huge range of measurement application domains demand revising and expanding the fundamentals of measurement, to develop a framework of general foundational concepts and principles universally recognized as valuable and effective in every field of empirical endeavor. This is confirmed by the fact that the problem of measurability is a key challenge in many knowledge domains outside of natural sciences where the development of appropriate measurement scales and processes is an actively disputed research topic [6]. A critical presentation of the complex events around the development of measurement in social sciences is in [7]. In addition, measurement science could and should have a crucial unifying 
and enabling role in the current mainstream efforts aimed at promoting the convergence of distinct disciplines underlying new broad fields of investigation. Unfortunately, multidisciplinary research on the foundations of measurement are very limited and rare, despite the fact that there is much more room for improvement in this field than in the investigation of highly specialized topics. Referring to this ubiquitous application scenario, this paper proposes some viewpoints and key issues on the motivation and the scope of measurement science.

\section{What is Measurement Science?}

The International Vocabulary of Metrology (VIM) defines metrology as the "science of measurement and its application” [8, 2.2]. That measurement is pervasively applied is not under discussion, but is there, properly speaking, a science of measurement? Or is measurement such a cross-disciplinary activity, from fundamental physics to psychology, that there are no actual commonalities or characterizing features identifiable for it? The point is not of neglecting the importance of measurement, whose effectiveness for experimental sciences is unanimously recognized and the only doubt remains whether measurement is merely a tool for them, even though a pivotal one, or a definitional element, as claimed by $\mathrm{N}$. R. Campbell, who stated that physics "might almost be described as the science of measurement” [9, p.267]. Rather, the issue we underscore here is about the nature and the disciplinary autonomy of the body of knowledge devoted to measurement.

Paradoxically, this doubt mainly arises in the context of physical measurement. Social sciences, lacking the well-established theoretical foundation that physics grants, have been eager to adopt and adapt measurement-related concepts and to give them a structural justification, thus generating a relevant literature around measurement scales (at least since [10]), measurement models (e.g., [11] and [12]), and finally measurement theory as such [13]. Physicists and engineers are sometimes unaware of this literature, for the generally good reason that the methods elaborated in social measurement - inclusively known as psychometrics [6] - are not so useful whenever measurement is a process whose experimental component includes a physical transducer. Hence, the paradox exists: physical measurement is much more effective than social measurement (or assessment: one questions even the measurability of non-physical properties and the issue has generated a long-standing debate [14], [15]). However, in recent decades, social scientists developed a reflection on foundations of measurement from which physicists and engineers could find something of interest. 
Were measurement science an autonomous science, it should be possible to exhibit its contents. What are they? The list of investigation fields of relevance for research in measurement is inherently multi-disciplinary: from solid-state physics and electronics, to systems theory and control theory, to signal theory and statistics, but also, information theory and computer science, biology and nanotechnologies, philosophy of science and ontology. In perspective, the supposition is that even political science and ethics will be progressively interested in measurement and its social implications. Is there a distinctive, common ground for a science of measurement in the diversity of these topics? In addition, even if it is accepted as a science, how would it be classified in reference to Popper's problem of demarcation [16]; as an experimental (as physics and chemistry) or a formal (as mathematics and logic) science? That is, does it generate experimentally falsifiable statements or theorems derivable from suitably chosen axioms? (Fig.1).

The answer to these questions is not univocal, although the value of specialization in academia and industry, so strongly emphasized in our epoch, would plausibly lead to the conclusion that measurement science is just a more or less fashionable term to denote an indistinct, fuzzy area of knowledge. A provocative opinion in this direction comes from the following quotation, attributed to E. Fermi: “There are two possible outcomes: if the result confirms the hypothesis, then you've made a measurement; if the result is contrary to the hypothesis, then you've made a discovery."

An objection could be that metrology is instead the niche area of super-high precision activities related to the definition of quantity units, their realization in primary standards and their key comparisons [17] as performed by National Metrology Institutes [18]. In fact, some contexts reverse the VIM definition and consider metrology this specific part of the broader body of knowledge devoted to measurement. Or, stating it another way, metrology might be characterized not by content, but by its emphasis on the organizational issues arising from that so peculiar sociotechnical infrastructure that is the metrological system. By focusing on measurement standards and traceability chains of calibrations aimed to guarantee the metrological traceability of measurement results and, more globally, the sustainability and the reliability of the international technical accreditation or certification systems, metrology is superposed to legal metrology.Measurement science would be then mainly a matter of emphasis. A researcher working on a physical effect is operating as a physicist. Then, in the role of a metrologist (s)he exploits the effect to design and build a transducer, (s)he studies the sensitivity, the resolution, the repeatability, etc., of the device, and finally (s)he calibrates the measuring instrument based on that transducer. 
All of these interpretations have both conceptual and historical justifications, but we believe that they still depict only a part, and not the most fundamental one, of the whole story. Not only is measurement becoming a connecting glue of our sociotechnical systems, but also measurement science is its conceptual ground. In what follows, we develop and argue this thesis.

\section{Reliably Connecting Worlds}

We can characterize measurement science fundamentally in respect to the mentioned problem of demarcation. Some disciplines like physics aim at the knowledge of aspects of the empirical world and some others like mathematics at the development of formal structures. Physics aims at producing theories of the empirical world that pass rigorous experimental tests and, because the theories pass the tests, physics considers the theories at least partially and temporarily true. Mathematics aims at producing theories that allow consistent demonstrability of interesting (in some sense of the word) theorems. However, despite this difference, or maybe exactly because of it, physics exploits mathematics as a tool to properly represent information and perform inference from it (consider the paradigmatic role of mathematics in physics). Mathematics looks for inspiration and experimental application in the context of physics (as in the case of the several sub-disciplines of applied mathematics, such as statistics and control theory). Hence, demarcation depicts two remarkably distinct realms - let us metaphorically consider them islands - which we call:

- $\quad$ empirical island and information island, if the emphasis is on the nature of the inhabitants (the distinction in atoms vs. bits is suggestive but misleading, because it does not catch the social and psychological phenomena belonging to the empirical island), and

- $\quad$ island of experimental methods and island of formal methods, if the emphasis is on exploration methods.

While the islands are distinct for inhabitants and exploration methods, bridges connect them: achievement of the goal of better knowing the whole world is by building and crossing bridges to and from the other island.

In the current understanding of human knowledge, refining the image further is possible by admitting the existence of an asymmetry:

- while the explorers of the information island might never desire to cross bridges (let us call them pure mathematicians or something like that; of course, they must exploit physical means to communicate their discoveries, but this is a practical, not a 
scientific, issue),

- in the exploration of the empirical island, the information contents and the formal methods have been proved more and more effective, as the status of physics witnesses as the most mathematized of all experimental sciences.

\section{A Different Viewpoint?}

Is this metaphor of two islands too sharp a separation? Do we not live in one world and so is there a distinction only on methods to know and intervene on it?

This is a venerable position that philosophers call monism (for a discussion on this concept, see [19]). It states, "everything is $\mathrm{X}$, where $\mathrm{X}$ is (depending on the author) matter, spirit, number, perception, etc.” While the present paper is surely not the right context to enter into such a complex discussion, if we just assume that:

- energy and information are fundamental entities, and

- they are not reducible to each other because energy is conserved and information is not, then the metaphor of the two islands becomes at least plausible.

An interesting and provocative viewpoint of the information island is

Physical reality is a disaster. It is way too complicated, and nothing is at all what is appears to be. [In physical reality] nothing can truly be measured. [...] Any measurement made in this universe is necessarily a rough approximation. [...] Mathematical reality, on the other hand, is imaginary. It can be as simple and pretty as I want it to be. I get to have all those perfect things I cannot have in real life. I will never hold a circle in my hand, but I can hold one in my mind. And I can measure it." [20].

In fact, purely empirical data are considered inaccessible [5], and the island explored by physics, chemistry, biology, ... (but also psychology, sociology, economy, ...) can only be known, and then described, by creating maps of it. But since maps are entities that belong to the other island, bridges are unavoidable, because they connect "the realm of things we say as distinguished from the realm of things we do” [21, p.226], and experimental sciences are aimed at producing things that can be said (and tested, and taught, and applied, etc.). 
can be thought of as the architecture of peculiarly reliable bridges, able to guarantee high quality maps of (portions of) the empirical island. However, where do these guarantees come from?

From the point of view of the information island, measurement results might be odd maps; hybrid mixtures of native entities, such as numbers, and more or less explicit references to entities belonging to the other island, called measurement standards, now literally widespread all around the world. In addition, entities like $1.234 \mathrm{~kg}$ have absolutely nothing in them sufficient to provide the mentioned guarantees of reliability. They can be easily created, e.g., by "guessing that the mass of this object is $1.234 \mathrm{~kg}$ " (a "things we say," indeed). That is, quantitative statements might be necessary in high quality maps (although ordinal scales of measurement make this claim questionable), but they are surely not sufficient, given that guesses, opinions, forecasts, judgments by experience, etc. can be stated in quantitative terms while nevertheless remaining arbitrary bad maps. Measurement science is not the science of quantity: measurement might be $a$, but surely not the, "process of assigning numbers to represent qualities” [9, p.267].

Hence, measurement results are high quality maps, thanks not to some intrinsic features of them, but because they are created by crossing high quality bridges. This is, in fact, a characterizing feature of measurement, and, therefore, of measurement science. In a pragmatic view, measurement can be defined [22], [23] as a process expected to be able to produce:

- information specific to the measurand, i.e., a given property of the object under measurement, then independent of any other property of the object or the surrounding environment (that is why sensitivity and selectivity of measuring instruments are so important). It is a condition about the appropriate attribution of information to its claimed object, and hence, a requirement of objectivity of information; and

- information that is interpretable in the same way by different subjects in different places and times because it is expressed in a form independent of the specific context and only refers to entities which are universally accessible. (This is clearly the case of $1.234 \mathrm{~kg}$, thanks to the definition of the kilogram measurement unit and the existence of effective traceability chains connecting daily usages and the realizations of the unit's definition.) It is a condition about the appropriate reconstruction of information by different subjects and hence, a requirement of inter-subjectivity of information. 


\section{Uncertainty as Quantification of Connection Reliability}

The analysis of the concrete feasibility of objective and inter-subjective information processes shows that they are not yes-no features.

There are certain human activities, which have apparently perfect sharpness. The realm of mathematics and of logic is such a realm, par excellence. Here we have yes-no sharpness - two numbers are either equal to each other or they are not; a certain point either lies on a given line or it does not; there is only one straight line connecting any two points. Now, it is a matter of observation that this yes-no sharpness is found only in the realm of things we say, as distinguished from the realm of things we do [21, p.226].

As a critical consequence, measurements and their results, which are maps of things we do, cannot be yes-no sharp: their goal is to be objective and inter-subjective; however, they cannot be completely. For sure, the basic target of measurement science is to develop empirical and formal tools for obtaining high quality maps. But, perfectly objective and intersubjective information is, generally, impossible to obtain for both epistemic and pragmatic reasons: it would require gathering an infinite quantity of information by means of an infinite quantity of resources. This leads to the conclusion that the two pillars of objectivity and intersubjectivity that construct the measurement science bridge lean on soft ground (Fig.2). Hence,

... when reporting the result of a measurement, it is obligatory that some quantitative indication of the quality of the result be given so that those who use it can assess its reliability [24, 0.1].

Until a relatively recent past, the existence of this soft ground was justified for purely experimental reasons. Metrologists assumed that quantities had intrinsic true values, that measurement could only estimate due to experimental errors. This is the conceptual basis of the distinction between systematic and random errors, which gave origin to the theory of errors and several more or less explicitly derived estimation and filtering techniques (techniques often grounded on further hypotheses, such as the linearity of the underlying phenomenon and its time stationarity, a precondition for repeatability of measurement and then statistical sampling). Accordingly, metrologists envisioned at least parts of the empirical island as having a true map although they did not know the map, only because the bridge for reaching the information island is not stable enough. 
Many different but converging reasons have made this understanding more and more alien to our culture, even though still implicitly widespread among some metrologists.

\section{Are Models Unavoidable?}

J. L. Borges beautifully argued about the relations between the world and its models in his short tale On Rigor in Science:

In that Empire, the Art of Cartography attained such Perfection that the map of a single Province occupied the entirety of a City, and the map of the Empire, the entirety of a Province. In time, those Unconscionable Maps no longer satisfied, and the Cartographers Guilds struck a Map of the Empire whose size was that of the Empire, and which coincided point for point with it. The following Generations, who were not so fond of the Study of Cartography as their Forebears had been, saw that that vast Map was Useless, and not without some Pitilessness was it, that they delivered it up to the Inclemencies of Sun and Winters. In the Deserts of the West, still today, there are Tattered Ruins of that Map, inhabited by Animals and Beggars; in all the Land there is no other Relic of the Disciplines of Geography [25].

The synthesis is that maps cannot be the territory [26], and this is not due to some flaws of the bridges connecting the two islands, but to the essential differences of such islands: the only true map would not be a map anymore, but the territory itself.

This has nothing to do with cultural relativism, as instead is sometimes mistakenly supposed. Measurement scientists are usually, and wisely, at least moderately realist and recognize as unproblematic that their instruments actually interact with parts of the territory, i.e., objects and their quantities, whose existence is independent of maps (i.e., models; we are omitting here any consideration of quantum measurement). On the other hand, the results of such interactions have to be maps (i.e., information entities) and not empirical objects. (The astute reader might look at the consequences of "a scheme for entirely abolishing all words whatsoever” in Gulliver's report of his visit to "the grand academy of Lagado” [27, part 3 chapter 5]). Hence, measurands (i.e., the quantities to which measurement results are attributed) are not intended by the VIM as “quantities subject to measurement” anymore [28, 
2.6]. Appropriately, the new definition is "quantities intended to be measured" [8, 2.3], where the presence of these intentions emphasizes the pragmatic knowledge-based nature of measurement and surely does not hinder realism, as clearly pointed out by M. Bunge. “An interpretive hypothesis, such as ' $e$ is the electron charge', involves the assumption that there are certain physical objects, e.g., electrons, that is, certain things out there, independent of the mind. [...] But this is an assumption that may turn to be false. Therefore one speaks of the hypothetical or intended referent of a theory — in the philosophical not the psychological sense of the word. Nonetheless a physical theory does talk, even though hypothetically, of real entities: total fictions are left to literature.” [29, p.58]. This is plausibly the most fundamental reason why the quality of the information conveyed by measurement is currently stated not in terms of measurement errors, i.e., differences with (generally unknowable) true values but of measurement uncertainty, formalizing the degree of belief attributed to the gathered information on the measurand [24], [30] - [33]. Measurement uncertainty is then an encompassing entity. It may take into account errors if identified, and, for some reason, not corrected, and more generally, encompass several different causes that prevent the complete objectivity and inter-subjectivity of measurement.

\section{Conclusions}

Measurement plays a fundamental role in sustaining a fair, efficient, and evolving society. However, despite its crucial importance, there is only a vague definition of the nature and extent of basic knowledge pertaining to measurement science, and there is not enough analysis or understanding of its multi-disciplinary fundamentals.

There can be many good historical reasons for this situation: mainly, those related to the problem of demarcation, as metrology shares features of both experimental and formal sciences and, therefore, it is neither a purely empirical nor a purely formal body of knowledge. In fact, while paradigmatically natural sciences build maps of the empirical island and mathematics studies how to build maps that populate the formal island, measurement science operates in between. This is its apparent weakness: considering bridges just as tools to make connections. However, this is also its strength; experimental sciences and their impact on practical human activities need such connections. Thus, we need a sharper definition and a deeper understanding of the measurement body of knowledge for an essential basis of multidisciplinary research and for the benefit of our society.

\section{Acknowledgement}

The authors are grateful to Mr. Damiano Zanchetta for allowing the use of his figures in this 
paper.

\section{References}

[1] W. Kula, Measures and Men, Princeton, NJ, USA: Princeton University Press, 1986.

[2] ISO 9000:2005, Quality management systems - Fundamentals and vocabulary, International Standardization Organization, 2005.

[3] BIPM, Evolving needs for metrology in trade, industry and society and the role of the BIPM Kaarls Report, Bureau International des Poids et Mesures, 2007. [Online]. Available: http://www.bipm.org/utils/en/pdf/Kaarls2007.pdf.

[4] K.R. Fowler and J. Schmalzel, “Why do we care about measurement?” IEEE Instr. and Meas. Mag., vol. 7, no. 1, pp. 38-46, 2004.

[5] T.S. Kuhn, “The function of measurement in modern physical sciences,” Isis, vol. 52, pp. 161-193, 1961.

[6] M. Wilson, "Using the concept of a measurement system to characterize measurement models used in psychometrics,” Measurement, vol. 46, no. 9, pp. 3766-3774, 2013.

[7] S.J. Gould, The Mismeasure of Man, New York, NY: W. W. Norton, 1981.

[8] JGCM 200:2012, International Vocabulary of Metrology - Basic and general concepts and associated terms (VIM), Joint Committee for Guides in Metrology, 2012. [Online].

Available: http://www.bipm.org/en/publications/guides/vim.html.

[9] N.R. Campbell, Physics - The Elements, Cambridge, England, UK: Cambridge University Press, 1920.

[10] S.S. Stevens, “On the theory of scales of measurement,” Science, vol. 103, no. 2684, pp. 677-680, 1946.

[11] L.L. Thurstone, “Attitudes can be measured,” American Journal of Sociology, vol. 33, no. 4, pp. 529-554, 1928.

[12] L. Guttman, “A basis for scaling qualitative data,” American Sociological Review, vol. 9, pp. 139-150, 1944.

[13] D. Krantz, R.D. Luce, P. Suppes, and A. Tversky, Foundations of Measurement, New York, NY, USA: Academic Press, 1990.

[14] J. Michell, Measurement in Psychology: A Critical History of a Methodological Concept, Cambridge, England, UK: Cambridge University Press, 1999.

[15] G.B. Rossi, “Measurability,” Measurement, vol. 40, pp. 545-562, 2007.

[16] S. Thornton, “Karl Popper”, The Stanford Encyclopedia of Philosophy (Spring 2013

Edition), E.N. Zalta, ed., [Online]. Available:

http://plato.stanford.edu/archives/spr2013/entries/popper.

[17] The BIPM key comparison database. [Online]. Available: http://kcdb.bipm.org. 
[18] National metrology institutes of Member States of the Metre Convention and Associate States and Economies of the CGPM, institutes designated in the CIPM MRA, and other institutes. [Online]. Available: http://www.bipm.org/en/practical_info/useful_links/nmi.html. [19] J. Schaffer, “Monism”, The Stanford Encyclopedia of Philosophy (Spring 2014 Edition), E.N. Zalta, ed., [Online]. Available: http://plato.stanford.edu/archives/spr2014/entries/monism. [20] P. Lockhart, Measurement, Cambridge, MA, USA: Harvard Univ. Press, 2012.

[21] P.W. Bridgman, “How much rigor is possible in physics?” in: L. Henkin, P. Suppes, and A. Tarski, eds., The Axiomatic Method, Amsterdam, Netherlands: North-Holland, 1959. [22] P. Carbone, L. Buglione, L. Mari, and D. Petri, “A comparison between foundations of metrology and software measurement,” IEEE Trans. Instr. Meas., vol. 57, no. 2, pp. 235-241, 2008.

[23] L. Mari, P. Carbone, and D. Petri, “Measurement fundamentals: a pragmatic view,” IEEE Trans. Instr. Meas., vol. 61, no. 8, pp. 2107-2115, 2012.

[24] JCGM 100:2008, Evaluation of measurement data - Guide to the expression of uncertainty in measurement (GUM, originally published in 1993), Joint Committee for guides in Metrology, 2008, [Online]. Available:

http://www.bipm.org/en/publications/guides/gum.html.

[25] J.L. Borges, Collected Fictions, New York, NY, USA: Penguin, 1999.

[26] G. Bateson, Steps to an Ecology of Mind, Chicago, IL, USA: University of Chicago Press, 1972.

[27] J. Swift, Gulliver's Travels, New York, NY, USA: Penguin Classics, 2003.

[28] ISO, International Vocabulary of Basic and General Terms in Metrology (VIM), second ed., International Standardization Organization, 1993.

[29] M. Bunge, Foundations of Physics, New York, NY, USA: Springer, 1967.

[30] A. Ferrero and S. Salicone, “Measurement uncertainty,” IEEE Instr. and Meas. Mag., vol. 9, no. 3, pp. 44-51, 2006.

[31] A. Ferrero and S. Salicone, "Uncertainty: only one mathematical approach to its evaluation and expression?”, IEEE Trans. Instr. Meas., vol. 61, no. 8, pp. 2167-2178, 2012. [32] W. Bich, "From errors to probability density functions: evolution of the concept of measurement uncertainty,” IEEE Trans. Instr. Meas., vol. 61, no. 8, pp. 2153-2159, 2012. [33] G. B. Rossi, “Toward an interdisciplinary probabilistic theory of measurement,” IEEE Trans. Instr. Meas., vol. 61, no. 8, pp. 2095-2106, 2012.

BIOS:

Luca Mari

Università Carlo Cattaneo (LIUC), 21053 Castellanza, Italy, e-mail: lmari@liuc.it 
Luca Mari (MS 1987; PhD 1994) is a Full Professor of measurement science with the Cattaneo University - LIUC, where he teaches courses on measurement science, statistical data analysis, system theory. He is currently the chairman of the TC1 (Terminology) and the secretary of the TC25 (Quantities and units) of the International Electrotechnical Commission (IEC), and an IEC expert in the WG2 (VIM) of the Joint Committee for Guides in Metrology (JCGM). He has been the chairman of the TC7 (Measurement Science) of the International Measurement Confederation (IMEKO).

\section{Dario Petri (dario.petri@unitn.it)}

University of Trento, 38123, Trento, Italy

Dario Petri is a Full Professor of measurement and electronic instrumentation at the Department of Industrial Engineering of the University of Trento. He is currently chairing the Italian Association of Electrical and Electronic Measurements (GMEE) and he is the Vice President for Finance of the IEEE Instrumentation and Measurement Society. Dr. Petri is the author of over 250 papers published in international journals or in proceedings of peer reviewed international conferences. Dario Petri is a Fellow member of IEEE.

\section{Figure captions;}

Fig.1. Scientific knowledge: the problem of demarcation and the role of measurement (C) 2014 Damiano Zanchetta, used with permission.)

Fig.2. Measurement as a bridge between the empirical island and the information island (C) 2014 Damiano Zanchetta, used with permission.)

\section{SUGGESTED HIGHLIGHTED STATEMENTS}

"Introduction":

Measurements are pervasively performed in a huge number of situations by a multitude of subjects. They are key enablers for the progress of our society.

The nature and extent of the body of knowledge pertaining to measurement are quite vaguely identified.

Measurement is becoming a connecting glue of our sociotechnical systems and measurement science is its conceptual ground. 
"What is measurement science?":

Measurement is a privileged tool for building bridges between the empirical and the information islands, or the island of experimental sciences and the island of formal sciences

"Uncertainty as quantification of bridge reliability":

In a pragmatic view, measurement can be defined as a both conceptual and empirical process able to produce information with a finite level of objectivity and intersubjectivity. 